\title{
Notoginsenoside Ft1 Promotes Fibroblast Proliferation via PI3K/Akt/mTOR Signaling Pathway and Benefits Wound Healing in Genetically Diabetic Mice ${ }^{\mathbb{S}}$
}

\author{
Eryun Zhang, Bo Gao, Li Yang, Xiaojun Wu, and Zhengtao Wang
}

Department of Pharmacognosy, China Pharmaceutical University, Nanjing, China (E.Z, Z.W.); and Shanghai Key Laboratory of Complex Prescriptions, Institute of Chinese Materia Medica, Shanghai University of Traditional Chinese Medicine, Shanghai, China (B.G., L.Y., X.W., Z.W.)

Received September 15, 2015; accepted November 12, 2015

\begin{abstract}
Wound healing requires the essential participation of fibroblasts, which is impaired in diabetic foot ulcers (DFU). Notoginsenoside Ft1 (Ft1), a saponin from Panax notoginseng, can enhance platelet aggregation by activating signaling network mediated through $\mathrm{P}_{2} \mathrm{Y}_{12}$ and induce proliferation, migration, and tube formation in cultured human umbilical vein endothelial cells. However, whether it can accelerate fibroblast proliferation and benefit wound healing, especially DFU, has not been elucidated. In the present study on human dermal fibroblast HDF-a, Ft1 increased cell proliferation and collagen production via PI3K/Akt/ mTOR signaling pathway. On the excisional wound splinting model established on $\mathrm{db} / \mathrm{db}$ diabetic mouse, topical application of Ft1 significantly shortened the wound closure time by 5.1 days in contrast with phosphate-buffered saline (PBS) treatment (15.8 versus 20.9 days). Meanwhile, Ft1 increased the rate of reepithelialization and the amount of granulation tissue at day 7
\end{abstract}

and day 14. The molecule also enhanced mRNA expressions of COL1A1, COL3A1, transforming growth factor (TGF)- $\beta 1$ and TGF- $\beta 3$ and fibronectin, the genes that contributed to collagen expression, fibroblast proliferation, and consequent scar formation. Moreover, Ft1 facilitated the neovascularization accompanied with elevated vascular endothelial growth factor, platelet-derived growth factor, and fibroblast growth factor at either mRNA or protein levels and alleviated the inflammation of infiltrated monocytes indicated by reduced tumor necrosis factor- $\alpha$ and interleukin- 6 mRNA expressions in the diabetic wounds. Altogether, these results indicated that Ft1 might accelerate diabetic wound healing by orchestrating multiple processes, including promoting fibroblast proliferation, enhancing angiogenesis, and attenuating inflammatory response, which provided a great potential application of it in clinics for patients with DFU.

\section{Introduction}

Wound healing is a well-orchestrated integration of complex biologic and molecular events, which requires the participation of many types of cells, including macrophages, fibroblasts, keratinocytes, and endothelial cells. During the process, fibroblasts play an important role by depositing extracellular matrix (ECM) that guides angiogenesis and supports the migration and proliferation of other cells that eventually form the scar (Martin, 1997; Falanga, 2005; Brem and Tomic-Canic, 2007; Gurtner et al., 2008). Accordingly, drugs targeting fibroblast proliferation may contribute to the skin wound healing. Indeed, as known so far, two engineered living skin

This work was supported financially by the National Natural Science Foundation of China [Grant 81530096], Key Research Innovation Project [Grant 13ZZ099], Key Project from Department of Education of China [Grant 20123107130002], Shanghai Three-Year Plan for Advancing Traditional Medicine [Grant ZY3-CCCX-3-3014] Shanghai Eastern Scholar Program [Grant 2013-59], and Shanghai E-research Institute of Bioactive Constituent in TCM plan.

dx.doi.org/10.1124/jpet.115.229369

S This article has supplemental material available at jpet.aspetjournals.org. products approved by the Food and Drug Administration containing allogeneic fibroblasts have been successfully applied in the therapy of patients with diabetic or venous skin ulcers (Griffith and Naughton, 2002).

Diabetic foot ulcers (DFUs) are characterized by disrupted wound healing process, which is estimated to occur in $15 \%$ of all patients with diabetes (Boulton et al., 2005) and $84 \%$ of all diabetes-related lower-leg amputations (Reiber et al., 1999). Patients of DFUs often suffer from expensive hospital costs and poor quality of life (Ramsey et al., 1999). A number of factors accounting for the nonhealing wounds in diabetes have been implicated, which include abnormal fibroblast migration, proliferation, differentiation, and apoptosis (Al-Mulla et al., 2011). Unfortunately, in the past few decades, little improvement has been made in preventing the morbidity and disability of DFU. The best available treatment of chronic wounds only achieves a $50 \%$ healing rate, often with temporary effect (Boulton et al., 2005). Therefore, more effective and specific drugs are urgently needed for the prevention or therapy of DFU.

ABBREVIATIONS: Akt, protein kinase B; DFU, diabetic foot ulcer; ECM, extracellular matrix; ELISA, enzyme-linked immunosorbent assay; FGF, fibroblast growth factors; Ft1, notoginsenoside Ft1; GT, granulation tissue; HDF, human dermal fibroblast; HE, hematoxylin/eosin; IL-6, interleukin6; mTOR, mammalian target of rapamycin; PDGF, platelet-derived growth factor; PI3K, phosphatidylinositol-3-kinase; $\alpha$-SMA, alpha-smooth muscle actin; TGF- $\beta$, transforming growth factor- $\beta$; TNF- $\alpha$, tumor necrosis factor- $\alpha$; VEGF, vascular endothelial growth factor. 


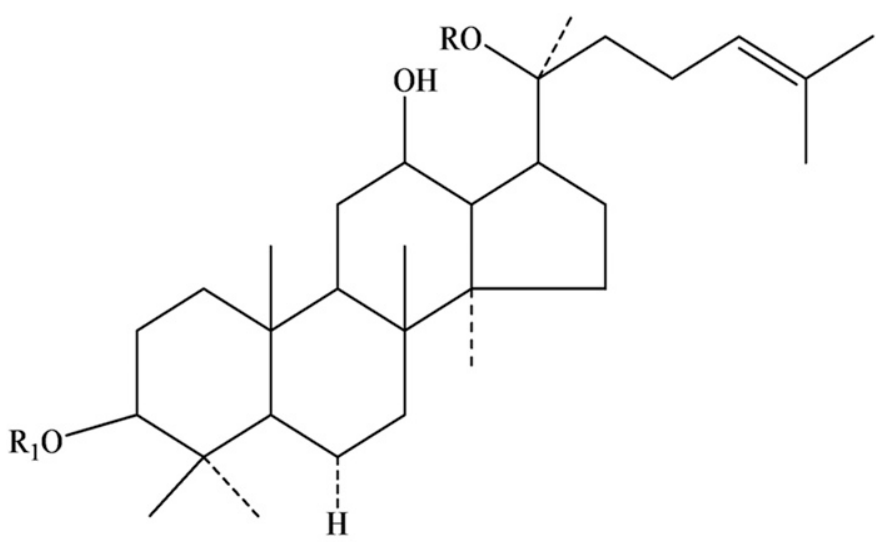

$$
R=H ; R 1=X y l 1-2 G l c 1-2 G l c
$$

Fig. 1. Chemical structure of Ft1.

Panax notoginseng (Burk.) F. H. Chen, known as Sanqi in China, is famous for its efficacy in treating trauma in East Asia over 2000 years (Chen, 1987). Notoginsenoside Ft1 (Ft1; Fig. 1), a saponin molecule, was first isolated from the leaves of $P$. notoginseng in 2006 (Chen et al., 2006). Our group previously reported that Ft1 was useful not only as a $\mathrm{P}_{2} \mathrm{Y}_{12}$ agonist in enhancing platelet aggregation (Gao et al., 2014) but also as a stimulator of proliferation, migration, and tube formation in cultured human umbilical vein endothelial cells (Shen et al., 2012). These two properties indicated its possible curative effect on wound healing. However, whether this molecule can promote the proliferation of fibroblast and benefit wound healing has not been demonstrated elsewhere.

The aim of this study was to investigate the effect of Ft1 on fibroblast proliferation and disclose the underlying mechanism. Moreover, its in vivo function on wound healing was assessed by an excisional wound splinting model established in genetically diabetic $(\mathrm{db} / \mathrm{db})$ mice. The research may contribute to the potential application of Ft1 in the therapy of DFU.

\section{Materials and Methods}

Chemicals and Regents. Ft1 was obtained from Shanghai R\&D Center for Standardization of Chinese Medicines (Shanghai, China). Its structure was confirmed using ${ }^{1} \mathrm{HNMR}$ and ${ }^{13} \mathrm{C}$ NMR spectral analysis, and its purity was more than $98 \%$ as determined by high pressure liquid chromatography analysis.

Trizol reagent was purchased from Life Technology (Carlsbad, CA). PrimeScript RT Master Mix and SYBR Premix Ex Taq were from Takara (Shiga, Japan). Phospho- PI3K p85 (Tyr458), PI3K, phosphoAkt (Thr308), Akt, phospho-mTOR (Ser2481) mTOR, and $\beta$-actin antibodies were obtained from Cell Signaling Technology (Danvers,
A

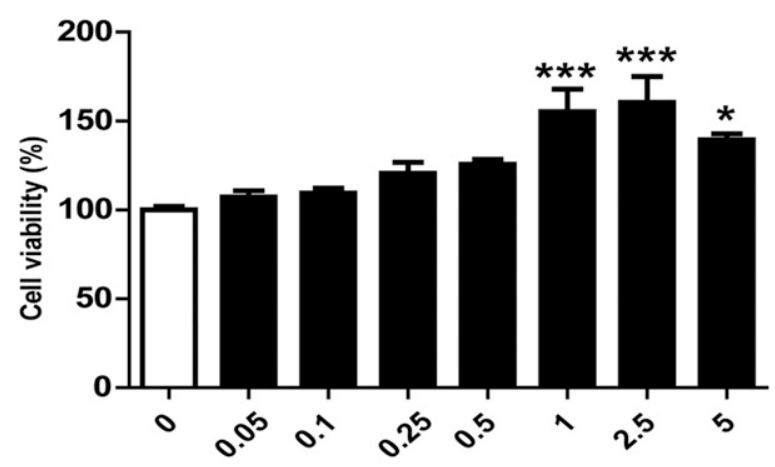

C

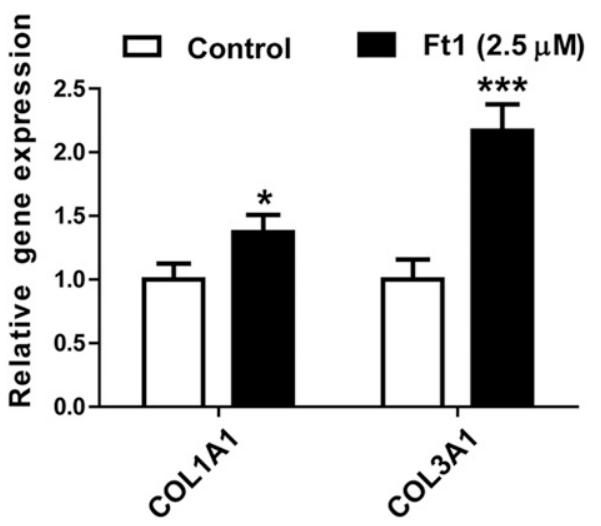

D

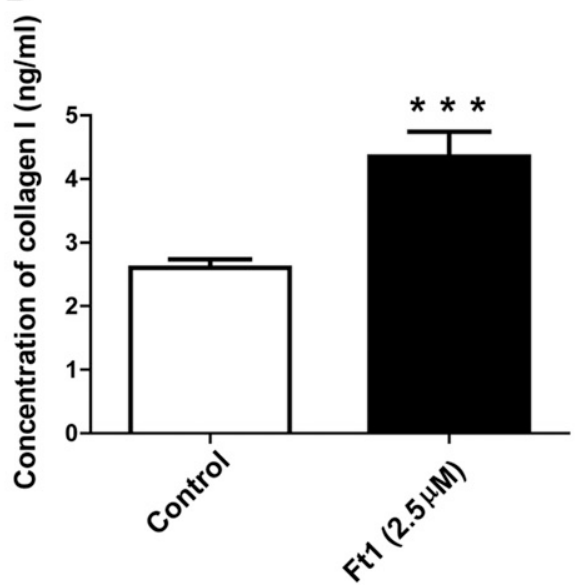

B

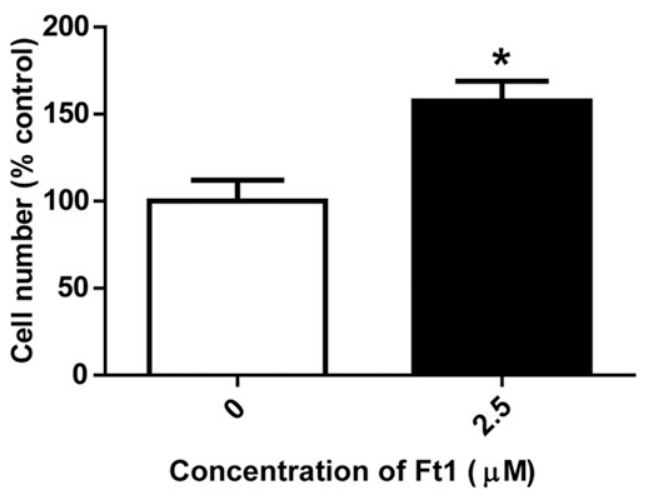

E

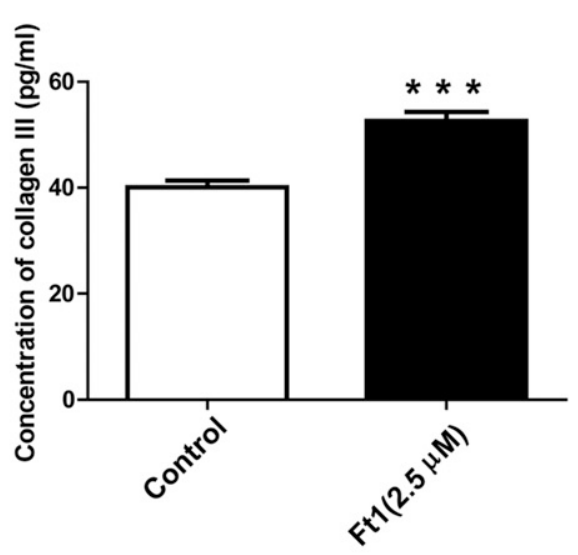

Fig. 2. Effects of Ft1 on cell proliferation and collagen production in HDF-a. (A and B) Ft1 induced cellular proliferation. (C) Ft1 increased gene expressions of COL1A1 and COL3A1. (D and E) Ft1 enhanced collagen I and collagen III production. Data were expressed as mean \pm S.E.M. $* P<0.05$ and $* * * P<0.001$ versus control, $n=6$. 
MA). Other antibodies against VEGF, CD31, and collagen III were provided by Abcam (Cambridge, UK). Pierce bicinchoninic acid protein assay kit was purchased from Thermo Fisher Scientific (Waltham, MA). Human recombinant vascular endothelial growth factor (VEGF) was supplied by PeproTech (Rocky Hill, NJ). Enzyme-linked immunosorbent assay (ELISA) kits were purchased from R\&D (Minneapolis, MN). All of the other reagents were from Sigma (St. Louis, MO) unless otherwise indicated.

Cell Culture and Cell Viability Measurement. Human dermal fibroblast-adult (HDF-a, ScienCell, San Diego, CA) cells were cultured in fibroblast medium (ScienCell) supplemented with $2 \%$ fetal bovine serum, $100 \mathrm{IU} / \mathrm{ml}$ penicillin, and $100 \mathrm{IU} / \mathrm{ml}$ streptomycin in a humidified atmosphere with $5 \% \mathrm{CO}_{2}$ at $37^{\circ} \mathrm{C}$. Cell viability after treatment with various concentrations of Ft1 for 48 hours was determined by MTT assay. Optical density was measured at $570 \mathrm{~nm}$ with $630 \mathrm{~nm}$ as the reference wavelength. Cell numbers were counted after trypsinizing HDF-a treated with Ft1 for 48 hours. Final cell viability and number of the treated cells were presented as the percentage to that of the control.

ELISA Analysis. After the cells were treated with Ft1 for 48 hours, the medium supernatant was collected and assayed for collagen I and III using commercial ELISA kits according to the manufacturer's instructions. The concentrations of the target proteins in the medium were determined with respective standard curves prepared using recombinant proteins of known concentrations.

Animal Model and Treatment. Female leptin receptordeficient (Lepr db/JNju, db/db) mice, 12 weeks old, with high blood glucose $(25.3 \pm 7.7 \mathrm{mM})$ were obtained from Nanjing Biomedical Research Institute of Nanjing University. The animals were singlehouse maintained under a 12 hour light/dark cycle at room temperature $\left(23 \pm 2^{\circ} \mathrm{C}\right)$ with free access to food and water. All animals received humane care according to the Institutional Animal Care guidelines approved by the Experimental Animal Ethical Committee of Shanghai University of Traditional Chinese Medicine. The excisional wound splinting model was generated according to the method described previously (Wang et al., 2013). In brief, after hair removal from the dorsal surface under anesthesia, two 6-mm fullthickness excisional skin wounds were created on mice at each side of the midline. A donut-shaped silicone splint was fixed around the wound with an immediate-bonding adhesive (Krazy Glue, Columbus, $\mathrm{OH})$. Each wound was treated with $15 \mu \mathrm{l}$ of Ft1 $(6.7 \mathrm{mg} / \mathrm{ml})$, vascular endothelial growth factor (VEGF) $(1.0 \mathrm{mg} / \mathrm{ml})$, or PBS every other day. After drug administration, the wound was covered with Tegaderm (Tegaderm, 3M, St. Paul, MN). The adhesive used was tested before the experiment, and no skin irritation or allergic reaction was observed.

Wound Analysis. Digital photographs of the wounds were taken on the day of surgery and every other day thereafter. Time to wound closure was defined as the time needed for the wound bed to be completely re-epithelialized and filled with new tissue. The pixel of the wound area was determined using Sigma Scan Pro Image Analysis Version 5.0 digital analysis software (Aspire Software International, Leesburg, VA) and was presented as the percentage of the initiative wound area. At day 7, 10,14, and 28, the mice were killed, respectively, and the wound skin samples including the surrounding skin $6 \mathrm{~mm}$ away were harvested using a 12-mm biopsy punch.

Histopathological Examination. For histologic preparations, the skin was fixed in $10 \%$ neutral buffered formalin and embedded in paraffin. Skin tissues were sectioned in $4-\mu \mathrm{m}$-thick slices for histopathological examination by hematoxylin/eosin (HE) staining and for collagen formation by Masson's trichrome staining.

For immunohistochemical staining, the sections were firstly incubated with $3 \% \mathrm{H}_{2} \mathrm{O}_{2}$ for 10 minutes to deactivate the endogenous peroxidase. To recover antigen, these sections were soaked in $10 \mathrm{mM}$ citrate buffer solution ( $\mathrm{pH}$ 6.0) and heated twice in the microwave oven. The slides were then washed thoroughly with PBS ( $\mathrm{pH} 7.4$ ). After blocked with $5 \%$ bovine serum albumin in Tris-buffered saline for 20 minutes, the sections were incubated with primary antibodies

A

$\mathrm{Ft} 1(2.5 \mu \mathrm{M})$

15

30

60 (min)

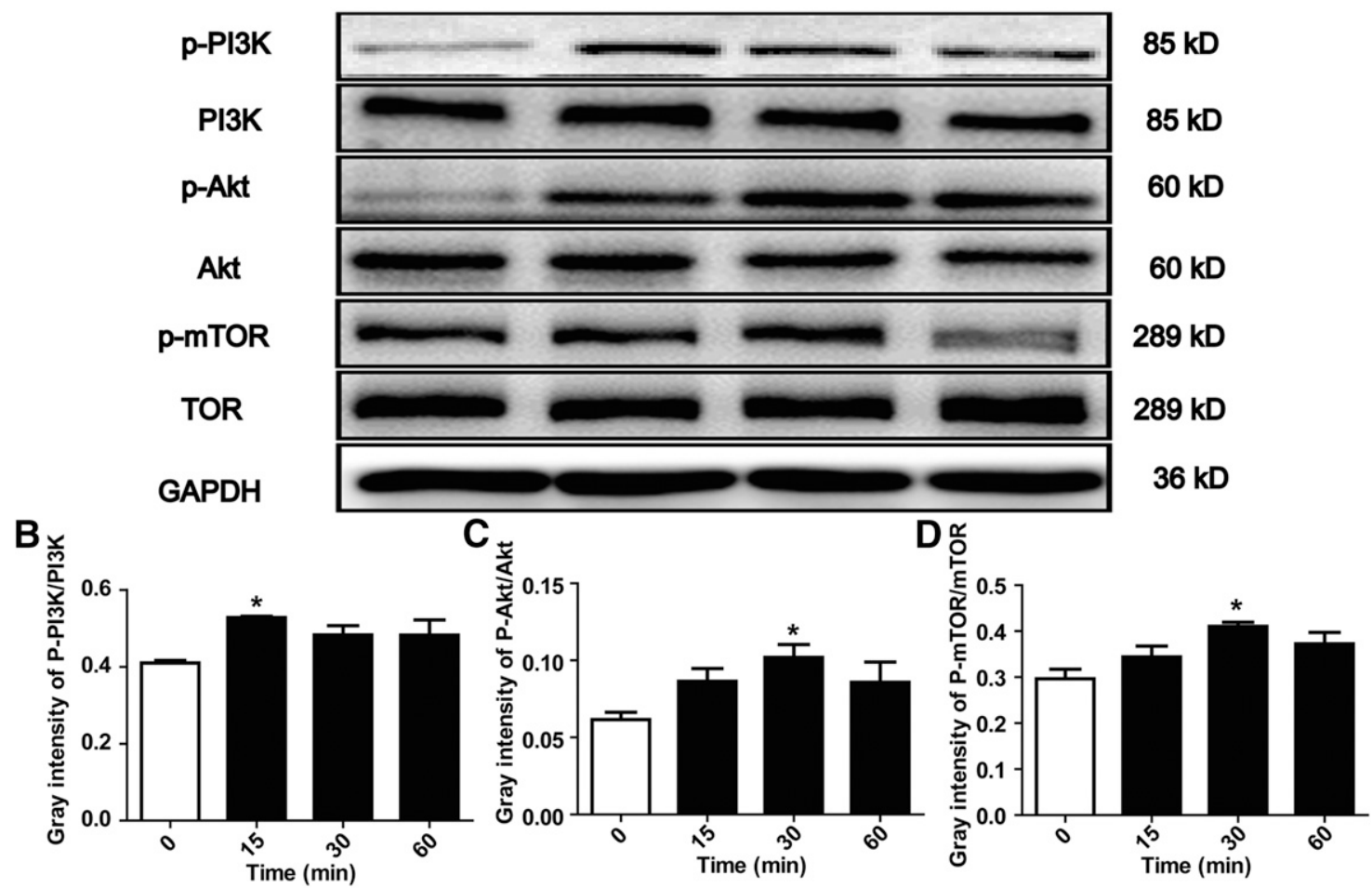

Fig. 3. Effect of Ft1 on the phosphorylation of PI3K/Akt/mTOR signaling pathway. Western blotting results showed that Ft1 treatment (2.5 $\mu \mathrm{M})$ time dependently activated PI3K, Akt, and mTOR on HDF-a cells. Data were expressed as mean \pm S.E.M. $* P<0.05$ versus control, $n=3$. 
against CD31 at $4^{\circ} \mathrm{C}$ overnight followed by thorough wash with PBS. Afterward, the slides were sequentially incubated with biotinylated secondary antibody for 20 minutes and streptavidin-horseradish peroxidase for another 20 minutes. The staining was visualized after incubation with a DAB- $\mathrm{H}_{2} \mathrm{O}_{2}$ solution. The slides were then counterstained with hematoxylin for 1 minute, dehydrated with ethanol, and sealed in resinene for microscopic observation.

Real-Time Polymerase Chain Reaction. Total RNA from cell and skin samples were isolated using Trizol reagent according to the manufacturer's instructions. cDNA was synthesized with PrimeScript RT Master Mix kit. Real-time polymerase chain reaction was performed with SYBR green premix in accordance with the manufacturer's instructions. The following primers were used for cDNA amplification: for COL1A1 (human), 5'-GCTACTACCGGGCTGATGAT-3' (forward) and 5'-ACCAGTCTCCATGTTGCAGA-3' (reverse); for COL3A1 (human), 5'-GAAGGGCAGGGAACAACTTG-3' (forward) and $5^{\prime}$-TTTGGCATGGTTCTGGCTTC-3' (reverse); for VEGF (human), 5'-CGCAGCTACTGCCATCCAAT -3' (forward) and 5'-CCCAC AGGGATTTTCTTGTCTT-3' (reverse); for FGF (human), 5'-GCCCAG TTCACTTCTTTGCA-3' (forward) and 5'-AGATCCAAACCCAGACCCAG-3' (reverse); for GAPDH (human), 5'-TGTTGCCATCAATGACC CCTT-3' (forward) and 5'-CTCCACGACFTACTCAGCG-3' (reverse); for VEGF (mouse), 5' -GCACATAGAGAGAATGAGCTTCC-3' (forward) and 5'-CTCCGCTCTGAACAAGGCT-3' (reverse); for PDGF (mouse), 5'-GAGGAAGCCGAGATACCCC-3' (forward) and 5' -TGC TGTGGATCTGACTTCGAG-3' (reverse); for FGF (mouse), 5'-ATGCT AGGGACCTGCCTTAGA-3' (forward) and 5'-AGCCAAGCAATGGGG AAGTG-3' (reverse); for TGF $\beta 1$ (mouse), $5^{\prime}$-CTCCCGTGGCTTCTAG TGC-3' (forward) and 5'-GCCTTAGTTTGGACAGGATCTG-3' (reverse); for TGF $\beta 3$ (mouse), 5'-CCTGGCCCTGCTGAACTTG-3' (forward) and 5' -TTGATGTGGCCGAAGTCCAAC-3' (reverse); for COL1 A1 (mouse), 5'-GCTCCTCTTAGGGGCCACT-3' (forward) and 5'-CCA CGTCTCACCATTGGGG-3' (reverse); for COL3A1 (mouse), 5'-CTGT AACATGGAAACTGGGGAAA-3' (forward) and 5'-CCATAGCTGAAC TGAAAACCACC-3' (reverse); for fibronectin (mouse), 5'-ATGTGGAC CCCTCCTGATAGT-3' (forward) and 5' -GCCCAGTGATTTCAGCAA AGG-3' (reverse); for TNF $\alpha$ (mouse), $5^{\prime}$-CCCTCACACTCAGATCATC TTCT-3' (forward) and 5'-GCTACGACGTGGGCTACAG-3' (reverse); for IL-6 (mouse), 5'-TAGTCCTTCCTACCCCAATTTCC-3' (forward) and 5'-TTGGTCCTTAGCCACTCCTTC-3' (reverse); for $\alpha$-SMA (mouse), 5'-AGGGAGTAATGGTTGGAATGG-3' (forward) and 5'-GG TGATGATGCCGTGTTCTA-3' (reverse); for $\beta$-actin (mouse), $5^{\prime}$-TGT CCACCTTCCAGCAGATGT-3' (forward) and 5'-AGCTCAGTAACAG TCCGCCTAGA-3' (reverse). All of the gene expressions were normalized to that of the internal reference genes, namely GAPDH or $\beta$-actin, within the same samples using the delta delta CT method.

Western Blotting Analysis. Cell and skin tissues homogenates were lysed in lysis buffer containing $50 \mathrm{mM}$ Tris (pH 7.5), 1 mM EDTA,
A

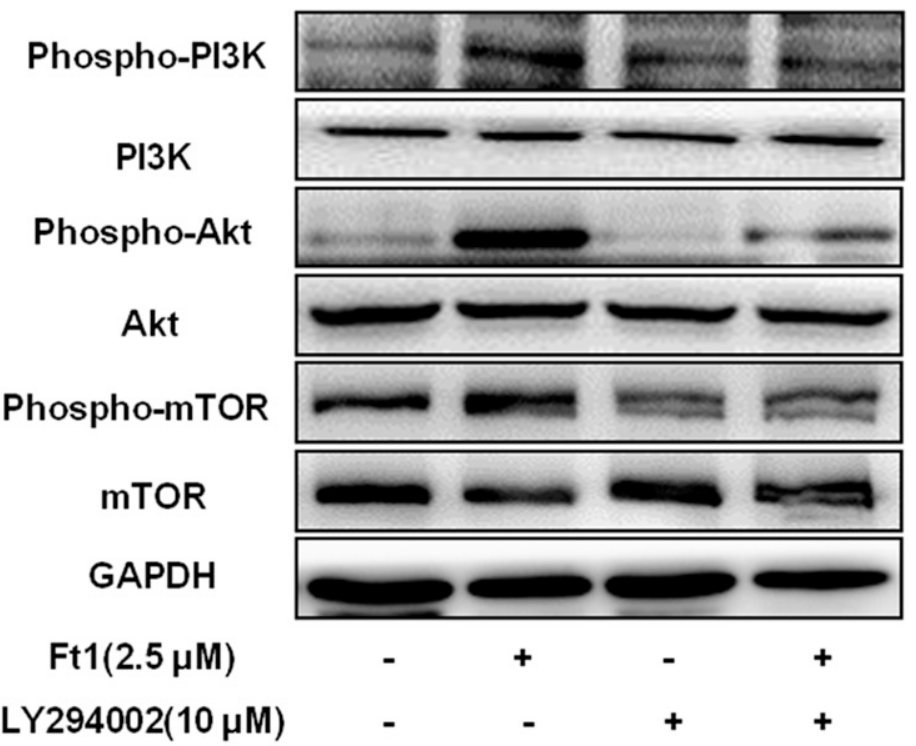

C

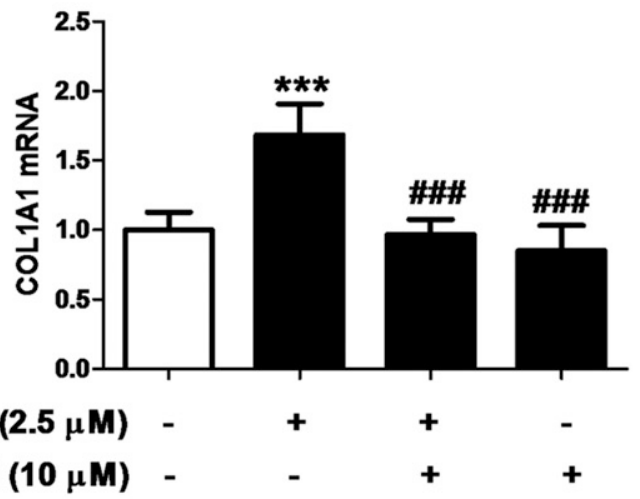

B
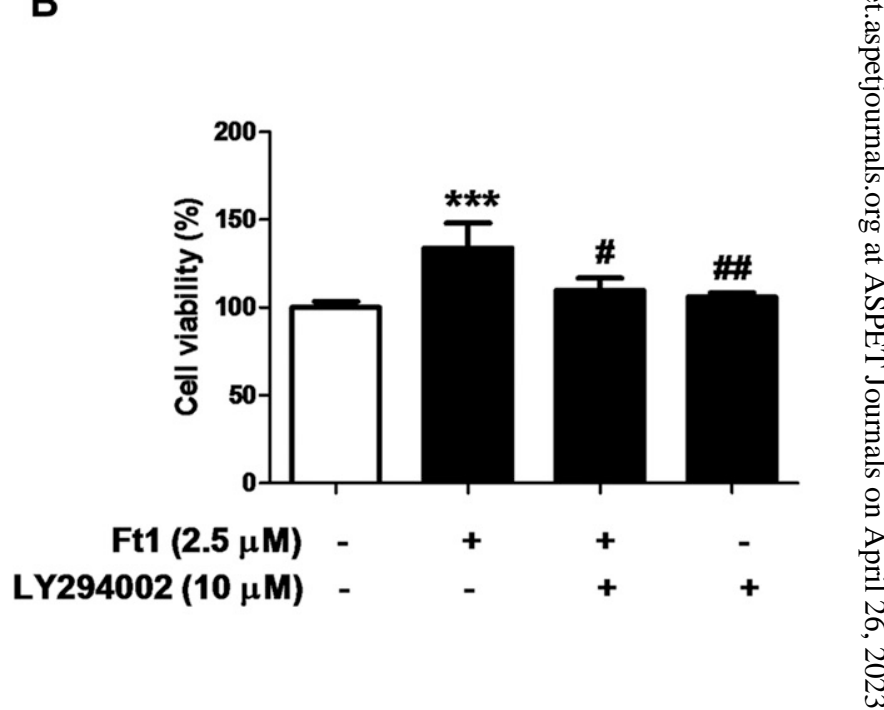

D

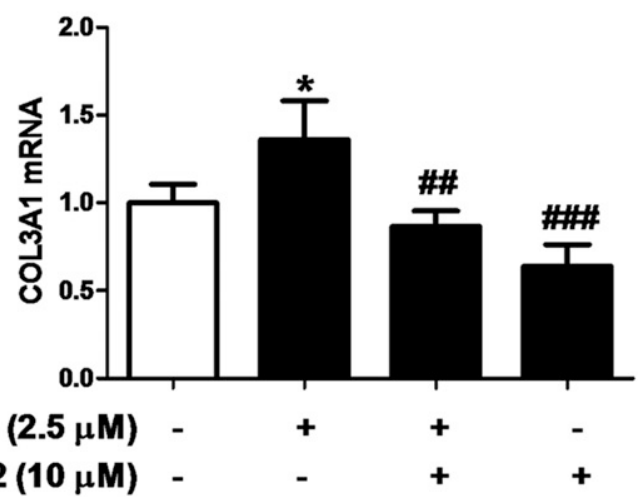

Fig. 4. Effect of LY294002 on cell proliferation and collagen mRNA expression of HDF-a cells. (A) Western blotting analysis of the phosphorylation of PI3K, Akt, and mTOR after Ft1 stimulation. (B) Cell proliferation measured after Ft1-stimulated for 48 hours. (C and D) mRNA expressions of COL1A1 and COL3A1 after Ft1 stimulation. All the cells were pretreated with LY294002 for 0.5 hour before the incubation with Ft1. Data were expressed as mean \pm S.E.M. $* P<0.05$, ${ }^{* * *} P<0.001$ versus control; $\# P<0.05$, \#\#P<0.01, and \#\#\#P<0.001 versus Ft1, $n=6$. 
$150 \mathrm{mM} \mathrm{NaCl}, 20 \mathrm{mM} \mathrm{NaF}, 0.5 \% \mathrm{NP}-40,10 \%$ glycerol, $1 \mathrm{mM}$ phenylmethylsulfonyl fluoride, $10 \mu \mathrm{g} / \mathrm{ml}$ aprotinin, $10 \mu \mathrm{g} / \mathrm{ml}$ leupeptin, and $10 \mu \mathrm{g} / \mathrm{ml}$ pepstatin A on ice. After centrifugation at $12,000 \mathrm{~g}$ for 15 minutes at $4^{\circ} \mathrm{C}$, the supernatant was collected, and its protein concentration was determined using bicinchoninic acid method. Total proteins, $40 \mu \mathrm{g}$ for each sample, were separated on $12 \%$ SDS-PAGE and transferred onto PVDF membranes (Millipore, Bedford, MA). After blocked with $5 \%$ bovine serum albumin in PBST $(0.1 \%$ Tween-20 in PBS) for 1 hour, the membranes were incubated with respective primary antibodies at $4^{\circ} \mathrm{C}$ overnight followed by a thorough wash with PBST. Thereafter, the membranes were incubated with horseradish peroxidase-conjugated secondary antibody (1:5000) for 1 hour at room temperature. The blots were developed by enhance chemiluminscence detection regents (GE Healthcare, Waukesha, WI). Gray intensity of protein bands was quantified with ImageJ and normalized to that of $\beta$-actin in each sample.
Statistical Analysis. All data were presented as mean \pm S.E.M. Difference among multiple groups was analyzed using one-way analysis of variance followed by Tukey's multiple comparison test with GraphPad Prism 5.0 software. Unpaired $t$ test was used to assess the difference between two groups. A value of $P<0.05$ was considered as statistically significant.

\section{Results}

Ft1 Induced Cellular Proliferation of HDF-a. Ft1 prompted the proliferation of HDF-a cells when used at the doses higher than $1 \mu \mathrm{M}$ (Fig. 2, A and B). Compared with the control, Ft1 used at $2.5 \mu \mathrm{M}$ increased the cell viability $(P<$ $0.001)$ and cell number $(P<0.05)$ of HDF-a by almost $50 \%$. Therefore this concentration was chosen for the successive experiments. After treatment for 24 hours, significant mRNA
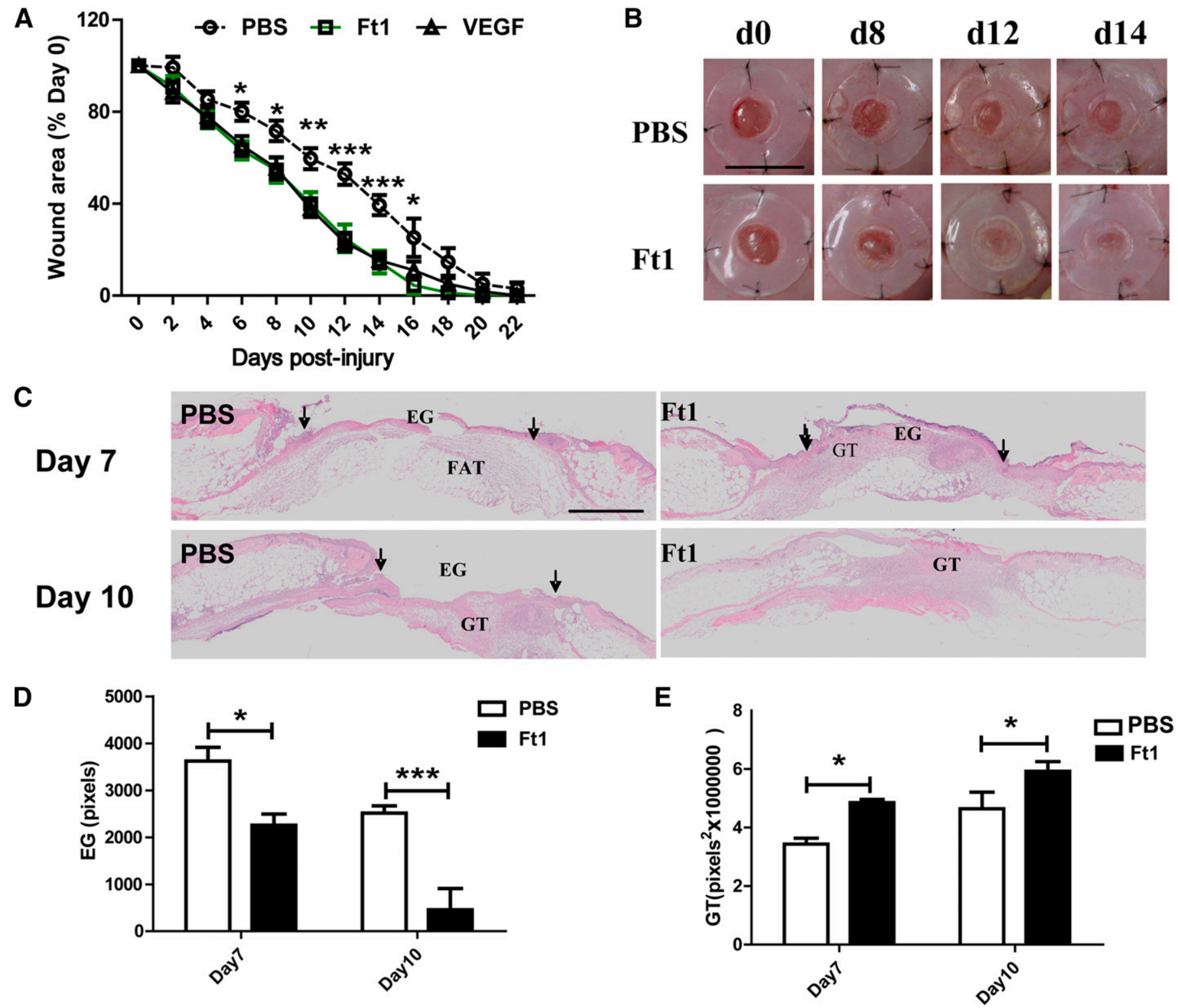

Fig. 5. Effect of Ft1 on wound closure in $\mathrm{db} / \mathrm{db}$ mice. (A) Change of wound area in 3 weeks. (B) Representative pictures of wounds at day $0,8,12,14$ postinjury. Scale bar $=10 \mathrm{~mm}$. (C) HE staining of diabetic mouse wounds at day 7 and 10 postinjury. Ft1-treated wounds closed earlier than the PBS-treated ones. Scale bar $=1 \mathrm{~mm}$. (D) Ft1 induced faster closure of epithelial gap and larger granulation tissue (GT). Results were presented as mean \pm S.E.M. $* P<0.05,{ }^{*} P<0.01$, and $* * * P<0.001$ versus control, $n=6$. EG, epithelial gap. 
A

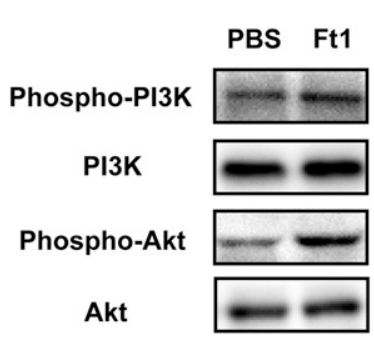

B

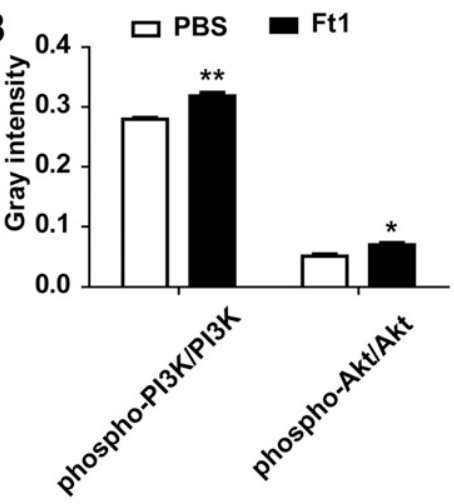

Fig. 6. Effect of Ft1 on the PI3K/Akt signaling pathway in the skin of diabetic animals at day 7 postinjury. (A) Western blotting assay. (B) Gray intensity analysis. The results showed that Ft1 treatment enhanced phosphorylation of PI3K and Akt in the wounds. Data were expressed as mean \pm S.E.M. ${ }^{*} P<0.05$ and ${ }^{* *} P<0.01$ versus control, $n=3$.

expressions of COL1A1 $(P<0.05)$ and COL3A1 $(P<0.001)$ was induced by Ft1 in HDF-a cells (Fig. 2C). In agreement with the mRNA expression pattern, Ft1 also increased the protein production of collagen I $(P<0.001)$ and collagen III
$(P<0.001)$ (Fig. 2, D and E). All of these results indicated the accelerative effect of $\mathrm{Ft} 1$ on the proliferation of fibroblast.

Ft1 Activated PI3K/Akt/mTOR Signaling Pathway in HDF-a. Upon stimulation by Ft1, PI3K, Akt, and mTOR were all activated in HDF-a as the phosphorylation of these signaling proteins were enhanced within 1 hour (Fig. 3, A and B). However, the phosphorylation peaks for these signaling molecules were reached at different time points. For PI3K, it seemed to be increasingly phosphorylated and reached its peak at 15 minutes $(P<0.05)$. For Akt and mTOR, both of them reached their phosphorylation peak at 30 minutes $(P<$ 0.05). To further confirm the involvement of PI3K/Akt/mTOR signaling pathway in Ft1-induced fibroblast proliferation, the PI3K inhibitor LY294002 was employed on HDF-a cells. As shown in Fig. 4A, LY294002 could efficiently abrogate the phosphorylation of PI3K as well as Akt and mTOR induced by Ft1. Accordingly, enhanced cell proliferation and COL1A1, COL3A1 mRNA expressions by Ft1 were all abolished by the chemical inhibitor (Fig. 4, B-D).

Ft1 Enhanced Wound Healing Process in Genetically Diabetic db/db Mice. Similar to VEGF, Ft1 accelerated wound closure in genetically diabetic $\mathrm{db} / \mathrm{db}$ mice. As shown in Fig. 5A, compared with PBS-treated wounds, topical Ft1
A

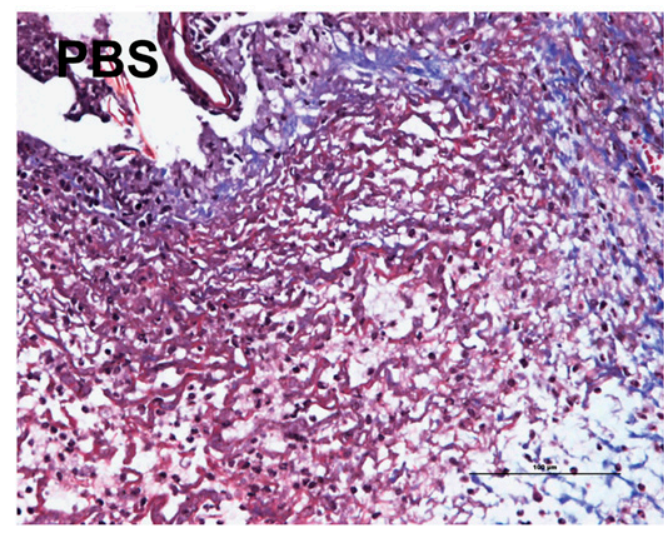

B

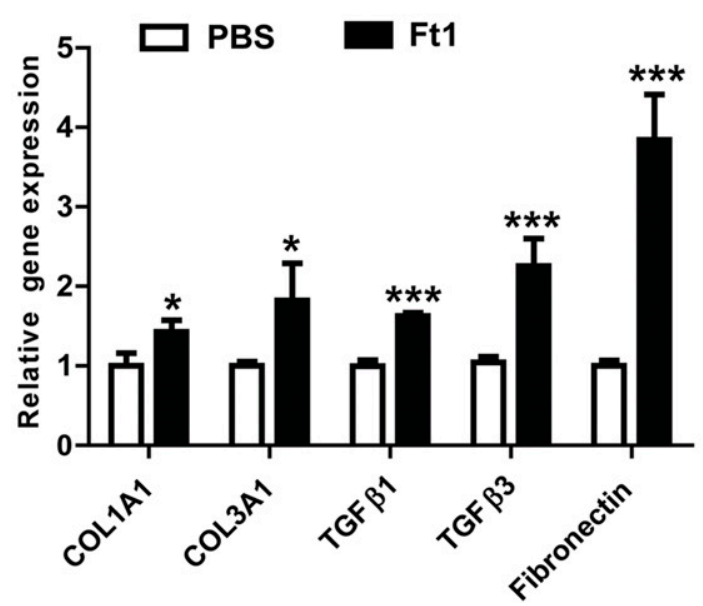

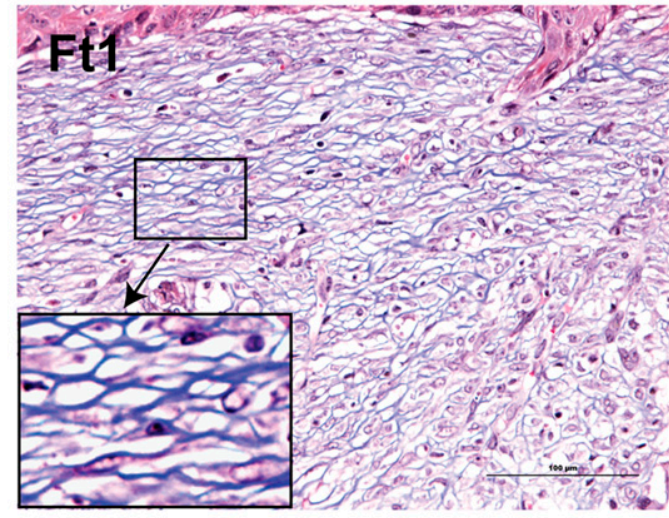

C PBS
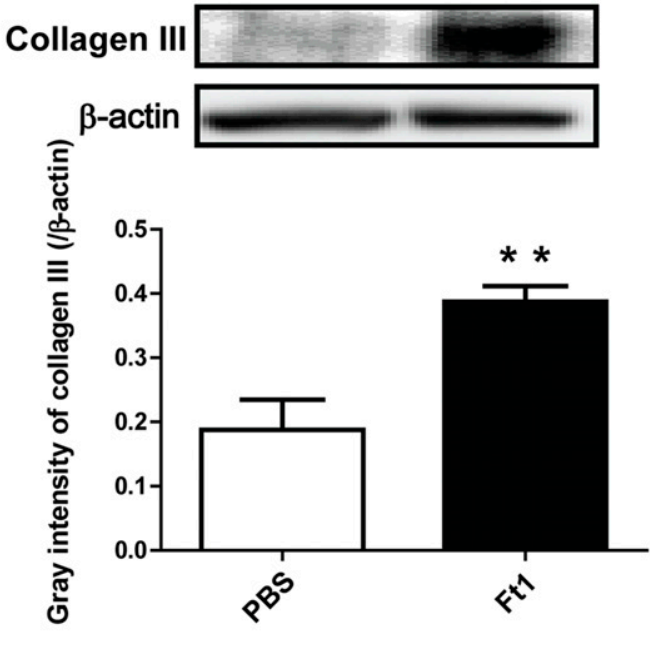

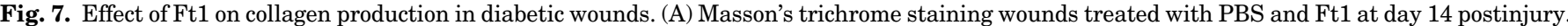

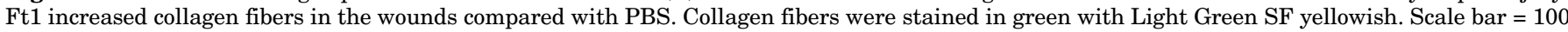

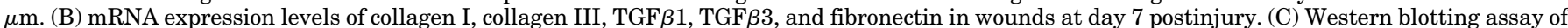

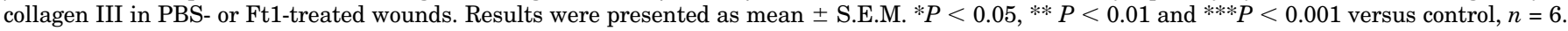


treatment significantly accelerated wound healing process. In diabetic mice, there was a significant decrease in terms of average wound area in Ft1-treated wounds in comparison with the PBS-treated control at day 6 postinjury $(P<0.05)$. On average, it took 15.8 days for Ft1-treated wounds to close completely in contrast with 20.9 days for PBS-treated controls. The increased healing rate of Ft1-treated wounds was clearly shown by the representative photographs at 8,12 , and 14 days postinjury (Fig. 5B), in which the epithelium of Ft1-treated wounds grew faster than that of PBS-treated controls at the same day.

Migration of the keratinocytes over the wounds for reepithelialization and ample granulation tissue formation are critical early markers of successful wound healing assessment (Trautmann et al., 2000). As shown in Fig. 5C, histologic assessment of diabetic wounds confirmed the increased healing rate of Ft1-treated wounds compared with PBS-treated controls. The re-epithelialization process of Ft1-treated wounds at day 7 and 10 postinjury was significantly faster than that of PBS-treated controls as demonstrated by reduced epithelial gaps in the Ft1-treated wounds (Fig. 5D; $P<0.05$ and $P<0.001$ ). Granulation tissue (GT), largely composed of fibroblasts synthesizing ECM proteins, in Ft1-treated wounds appeared to be thicker and larger. Quantitative measurement of the GT area of the wounds exposed that Ft1 treatment significantly promoted GT growth at day 7 and day 14 postinjury (Fig. 5E; $P<0.05$ ).

Consistent with its effect on PI3K/Akt signaling pathway in HDF-a cells, Ft1 treatment enhanced phosphorylation of PI3K $(P<0.01)$ and Akt $(P<0.05)$ significantly in the skin of diabetic animals at day 7 postinjury (Fig. 6, A and B). Masson's trichrome staining exposed that Ft1 promoted collagen production in the wounds (Fig. 7A) as more collagen fibers stained in blue and regularly arranged could be found in Ft1-treated wounds at day 14 postinjury compared with PBS-treated ones. Consistently, protein expression level of collagen III in Ft1treated wound tissues was higher than that in the controls (Fig. 7C; $P<0.05$ ). mRNA expression levels of proteins such as COL1A1, COL3A1, TGF $\beta 1$, TGF $\beta 3$, and fibronectin that had been reported to contribute to collagen formation were all elevated markedly (Fig. 7B; $P<0.05$ or $P<0.001$, respectively).

Because neovascularization is also important for wound repair, capillary formation was then examined in the wounds.
A

Day 7
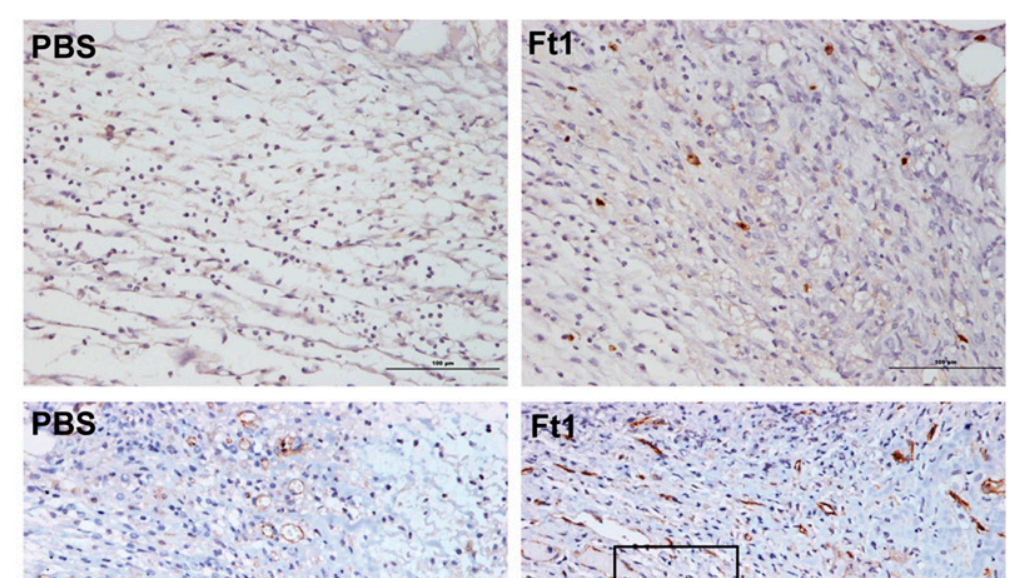

Day 14
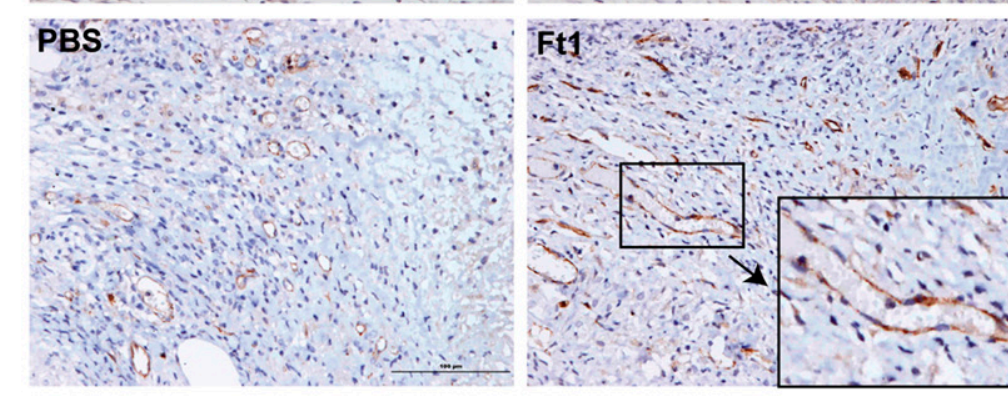

B

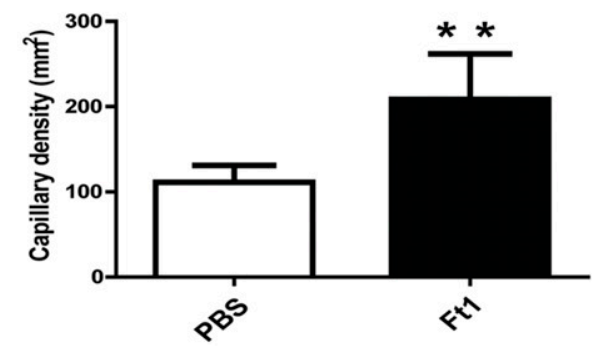

D

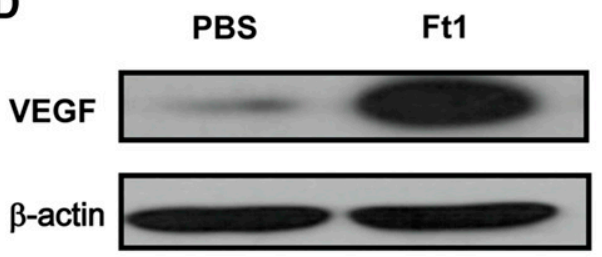

\section{C}
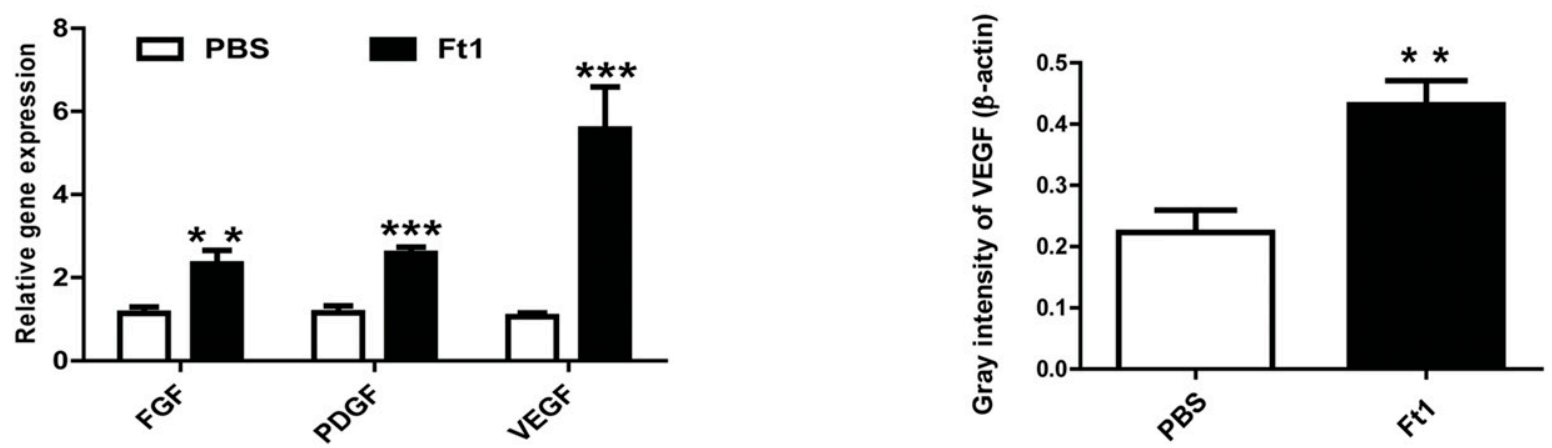

Fig. 8. Effect of Ft1 on neovascularization in diabetic wounds. (A) Immunostaining for CD31 at day 7 and 14 postinjury. Scale bar $=100 \mu \mathrm{M}$. (B) Capillary density at day 14 postinjury. (C) mRNA expression levels of FGF, PDGF, and VEGF in wounds at day 7 post-injury. (D) Western blotting assay of VEGF at day 7 postinjury. Results were presented as mean \pm S.E.M. $* * P<0.01$ and $* * * P<0.001$ versus control, $n=6$. 
Surprisingly, Ft1 treatment contributed to the capillary formation at day 14 postinjury as more CD31-positive capillaries were found in the wounds treated by Ft1 (Fig. 8A), which was further corroborated by capillary densities assessment (Fig. 8B; $P<0.01$ ). In addition, mRNA expression levels of FGF, PDGF, and VEGF as well as protein expression level of VEGF were remarkably elevated in Ft1-treated wounds compared with PBS-treated ones (Fig. 8, C and D; $P<0.01$ or $P<0.001$ ).

Moreover, HE staining exposed that less infiltration of monocytes was found in Ft1-treated wounds (Fig. 9A). Accordingly mRNA expressions of inflammatory cytokines such as TNF- $\alpha$ and IL- 6 were significantly decreased in the skin of diabetic animals at day 7 postinjury (Fig. 9B; $P<0.05$ or $P<0.001$ ).
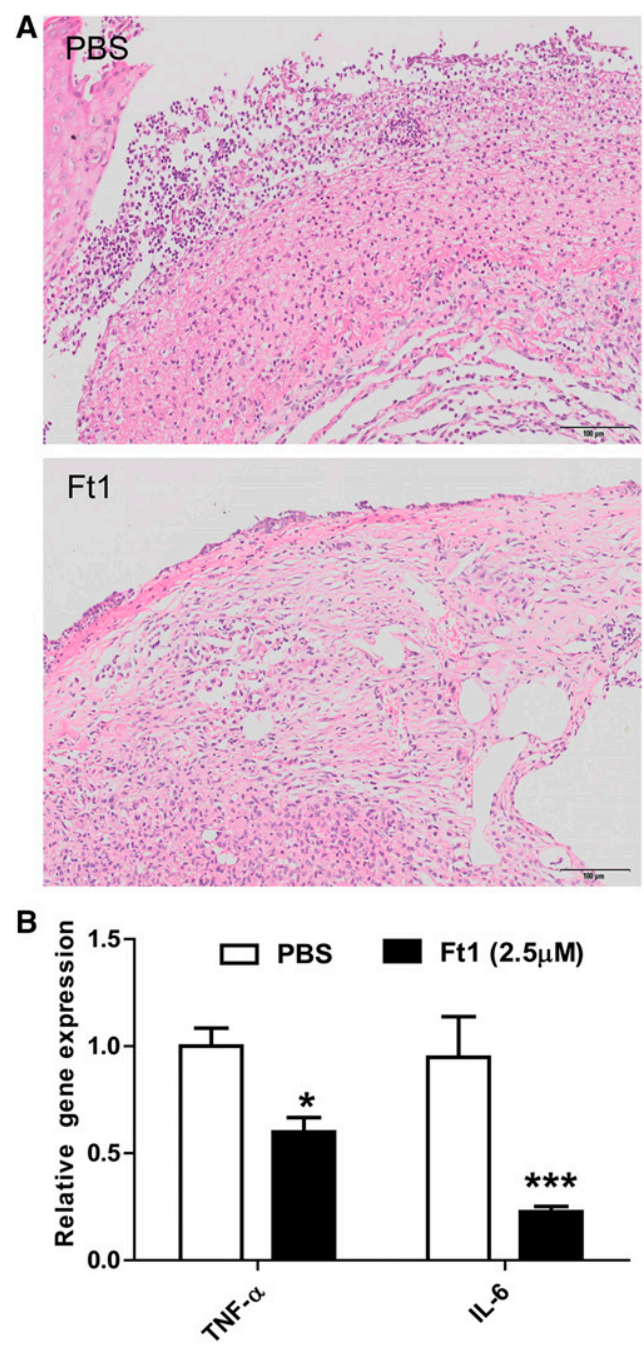

Fig. 9. Effect of Ft1 on inflammation in diabetic wounds. (A) HE staining of diabetic mouse wounds at day 7 postinjury. Ft1-treated wounds exhibited the less of infiltration of monocytes. Scale bar $=100 \mu \mathrm{m}$. (B) mRNA expression levels of TNF- $\alpha$ and IL- 6 in wounds at day 7 postinjury. Results were presented as mean \pm S.E.M. ${ }^{*} P<0.05$ and ${ }^{* * *} P<0.001$ versus control, $n=6$.

\section{Discussion}

Wound healing is a complex, dynamic, and orderly controlled process that involves three different but overlapping phases (Gurtner et al., 2008), namely inflammation, new tissue formation, and remodeling. Tissue injury triggers acute inflammatory response, in which neutrophils, monocytes, and mast cells infiltrate the site of injury and produce cytokines. The released cytokines then stimulate the proliferation and migration of several kinds of cells such as keratinocytes, endothelial cells, and fibroblasts to the wound. In the last step, extracellular matrix remodeling, angiogenesis, and reepithelialization are responsible for the wound closure and scar formation (Trautmann et al., 2000; Gurtner et al., 2008; Peppa et al., 2009). Abnormal pathology in DFU, including microvascular disease, peripheral neuropathy, decreased growth factor production, chronic hypoxia, hyperglycemia, and impaired angiogenesis (Reiber et al., 1999), delays wound healing or leads to the nonhealing wounds, which is further aggravated due to lack of effective treatments. Here, we provided compelling evidence demonstrating that Ft1 accelerated fibroblast proliferation and wound healing in diabetic mice, suggesting promising application of the compound in the therapy of DFU.

In the current study, an excisional wound splinting model was established in which the splint was tightly adhered to the skin around the wound, resulting in uniform wound closure by minimizing variations due to skin contraction and wound dressings (Wang et al., 2013). The wound, therefore, healed through granulation and re-epithelialization, a process similar to that occurring in humans (Galiano et al., 2004). To assess the effect of Ft1 on diabetic wounds, genetically diabetic $\mathrm{db} / \mathrm{db}$ mice were used, which has been proven to be an efficient model to test new agents for the therapy of DFU (Jacobi et al., 2002; Galeano et al., 2004). Our results indicated that topical application of Ft1 significantly promoted the wound healing process, including epidermal regeneration, and formation of granulation tissue and new well-structured capillary vessels.

Fibroblasts are the major cells found in the granulation of wound tissues that responsible for most collagen synthesis and organization of the ECM components (Mansbridge et al., 1999). They play an essential role in wound healing, including secretion of a series of growth factors that facilitates angiogenesis and matrix deposition (Beldon, 2010; Al-Mulla et al., 2011). Some of the fibroblasts in the injured tissue may differentiate into a highly contractile phenotype, i.e., myofibroblasts (Ross et al., 1970; Gabbiani, 1996),which have bundles of $\alpha$-smooth muscle actin (SMA) contributing to the closure of wound (Gabbiani, 2003; Lanning et al., 2000). Collagen and fibronectin are important components of granulation tissue, contributing to its integrity and delivery of tethered growth factors (Park et al., 2005; Hamed et al., 2011). Proteins in TGF- $\beta$ family attract macrophages into the wound area and stimulate them to produce additional cytokines, which further enhance fibroblast and smooth muscle chemotaxis and modulate collagen expression as well as consequent scar formation (Beldon, 2010). Our results showed that Ft1 treatment could increase mRNA expressions of $\alpha$-SMA (Supplemental Fig. 1; $P<0.01$ ), fibronectin, collagen (COL1A1, COL3A1), and TGF- $\beta$ (TGF- $\beta 1$ and TGF- $\beta 3$ ) in excisional wounds of $\mathrm{db} / \mathrm{db}$ mice. In human fibroblast cell HDF-a, Ft1 
also could enhance mRNA expressions of COL1A1 and COL3A1 as well as protein expressions of collagen I and III, which was mediated by PI3K/Akt/mTOR signaling pathway. Our findings suggested that Ft1 stimulated fibroblast proliferation and myofibroblast differentiation in the wound and thus accelerated wound closure.

Neovascularization is considered to play a crucial pathophysiological role in wound repair (Martin, 1997; Falanga, 2005; Gurtner et al., 2008). The formation of new blood vessels is essential to nourish the newly formed granulation tissue and the survival of keratinocytes. Our previous study showed that Ft1 induces proliferation, migration, and tube formation of cultured human umbilical vein endothelial cells (Shen et al., 2012), which are used extensively as an in vitro model for angiogenesis research (Park et al., 2006). The proliferation, migration, and formation of tubular structure of endothelial cells are the indications for the development of new blood vessels from pre-existing vascular bed in angiogenesis (Holash et al., 1999; Lamalice et al., 2007). In this study, Ft1-treated wounds enhanced capillary density, suggesting that Ft1 boosted local vessel growth in the wounds. Notably, Ft1 induced significantly increased levels of proangiogenic molecules, such as VEGF, PDGF, and bFGF, in the wounds or in HDF-a cells (Supplemental Fig. $2 ; P<0.05$ ), which might be partially responsible for its wound-healing function.

Excessive inflammation, associated with a prolonged persistence of neutrophil infiltration, is a consistent feature of diabetes-impaired wound healing (Nussler and Billiar, 1993). Some agents with an inhibitory ability in response to the inflammation, such as EPO, were shown to be effective in treating diabetic wounds (Eming et al., 2007). In the present study, topical Ft1 treatment could reduce infiltration of monocytes and suppress the proinflammatory cytokines, namely IL- 6 and TNF- $\alpha$, in diabetic wound beds, implying an inhibitory effect on the inflammation in diabetic wounds.

In summary, we demonstrated that Ft1 enhanced fibroblast proliferation via activating of PI3K/Akt/mTOR signaling pathway. Moreover, Ft1 accelerated wound healing in diabetic mice by orchestrating multifaceted factors in promoting reepithelialization, granulation tissue formation, synthesis of collagen, angiogenesis, and preventing excessive inflammatory response. All of these results suggested potential application of Ft1 in the clinical therapy of DFU.

\section{Acknowledgments}

The authors thank all members of Dr. Wang's, Dr. Wu's, and Dr. Yang's laboratories at Shanghai University of Traditional Chinese Medicine for their helpful feedback and suggestions.

\section{Authorship Contributions:}

Participated in research design: Zhang, Gao, Yang, Wu, and Wang. Conducted experiments: Zhang and Gao.

Performed data analysis: Zhang and Gao.

Wrote or contributed to the writing of the manuscript: Zhang, Yang, $\mathrm{Wu}$, and Wang.

\section{References}

Al-Mulla F, Leibovich SJ, Francis IM, and Bitar MS (2011) Impaired TGF- $\beta$ signaling and a defect in resolution of inflammation contribute to delayed wound healing in a female rat model of type 2 diabetes. Mol Biosyst 7:3006-3020.

Beldon P (2010) Basic science of wound healing. Surgery 28:409-412.

Boulton AJM, Vileikyte L, Ragnarson-Tennvall G, and Apelqvist J (2005) The global burden of diabetic foot disease. Lancet 366:1719-1724.
Brem H and Tomic-Canic M (2007) Cellular and molecular basis of wound healing in diabetes. J Clin Invest 117:1219-1222.

Chen JT, Li HZ, Wang D, Zhang YJ, and Yang CR (2006) New dammarane monodesmosides from the acidic deglycosylation of notoginseng-leaf saponins. Helv Chim Acta 89:1442-1448.

Chen QS (1987) [Pharmacological studies on notoginseng saponins isolated from the fibrous root of Panax notoginseng]. Zhong Yao Tong Bao 12:45-47.

Eming SA, Krieg T, and Davidson JM (2007) Inflammation in wound repair: molecular and cellular mechanisms. J Invest Dermatol 127:514-525.

Falanga V (2005) Wound healing and its impairment in the diabetic foot. Lancet $\mathbf{3 6 6}$ $1736-1743$.

Gabbiani G (1996) The cellular derivation and the life span of the myofibroblast. Pathol Res Pract 192:708-711.

Gabbiani G (2003) The myofibroblast in wound healing and fibrocontractive diseases. J Pathol 200:500-503.

Galeano M, Altavilla D, Cucinotta D, Russo GT, Calò M, Bitto A, Marini H, Marini R, Adamo EB, and Seminara P, et al. (2004) Recombinant human erythropoietin stimulates angiogenesis and wound healing in the genetically diabetic mouse. Diabetes 53:2509-2517.

Galiano RD, Michaels J, 5th, Dobryansky M, Levine JP, and Gurtner GC (2004) Quantitative and reproducible murine model of excisional wound healing. Wound Repair Regen 12:485-492.

Gao B, Huang L, Liu H, Wu H, Zhang E, Yang L, Wu X, and Wang Z (2014) Platelet $\mathrm{P}_{2} \mathrm{Y}_{12}$ receptors are involved in the haemostatic effect of notoginsenoside Ft1, a saponin isolated from Panax notoginseng. $\mathrm{Br} J$ Pharmacol 171:214-223.

Griffith LG and Naughton G (2002) Tissue engineering-current challenges and expanding opportunities. Science 295:1009-1014.

Gurtner GC, Werner S, Barrandon Y, and Longaker MT (2008) Wound repair and regeneration. Nature 453:314-321.

Hamed S, Ullmann Y, Egozi D, Daod E, Hellou E, Ashkar M, Gilhar A, and Teot L (2011) Fibronectin potentiates topical erythropoietin-induced wound repair in diabetic mice. J Invest Dermatol 131:1365-1374.

Holash J, Wiegand SJ, and Yancopoulos GD (1999) New model of tumor angiogenesis: dynamic balance between vessel regression and growth mediated by angiopoietins and VEGF. Oncogene 18:5356-5362.

Lamalice L, Le Boeuf F, and Huot J (2007) Endothelial cell migration during angiogenesis. Circ Res 100:782-794.

Lanning DA, Diegelmann RF, Yager DR, Wallace ML, Bagwell CE, and Haynes JH (2000) Myofibroblast induction with transforming growth factor-beta1 and -beta3 in cutaneous fetal excisional wounds. J Pediatr Surg 35:183-188.

Jacobi J, Jang JJ, Sundram U, Dayoub H, Fajardo LF, and Cooke JP (2002) Nicotine accelerates angiogenesis and wound healing in genetically diabetic mice. Am J Pathol 161:97-104.

Mansbridge JN, Liu K, Pinney RE, Patch R, Ratcliffe A, and Naughton GK (1999) Growth factors secreted by fibroblasts: role in healing diabetic foot ulcers. Diabetes Obes Metab 1:265-279.

Martin P (1997) Wound healing-aiming for perfect skin regeneration. Science 276 75-81.

Nussler AK and Billiar TR (1993) Inflammation, immunoregulation, and inducible nitric oxide synthase. J Leukoc Biol 54:171-178.

Park HJ, Zhang Y, Georgescu SP, Johnson KL, Kong D, and Galper JB (2006) Human umbilical vein endothelial cells and human dermal microvascular endothelial cells offer new insights into the relationship between lipid metabolism and angiogenesis. Stem Cell Rev 2:93-102.

Park SG, Shin H, Shin YK, Lee Y, Choi EC, Park BJ, and Kim S (2005) The novel cytokine p43 stimulates dermal fibroblast proliferation and wound repair. Am J Pathol 166:387-398.

Peppa M, Stavroulakis P, and Raptis SA (2009) Advanced glycoxidation products and impaired diabetic wound healing. Wound Repair Regen 17:461-472.

Ramsey SD, Newton K, Blough D, McCulloch DK, Sandhu N, Reiber GE, and Wagner EH (1999) Incidence, outcomes, and cost of foot ulcers in patients with diabetes. Diabetes Care 22:382-387.

Reiber GE, Vileikyte L, Boyko EJ, del Aguila M, Smith DG, Lavery LA, and Boulton AJ (1999) Causal pathways for incident lower-extremity ulcers in patients with diabetes from two settings. Diabetes Care 22:157-162.

Ross R, Everett NB, and Tyler R (1970) Wound healing and collagen formation. VI. The origin of the wound fibroblast studied in parabiosis. J Cell Biol 44: $645-654$.

Shen K, Ji L, Gong C, Ma Y, Yang L, Fan Y, Hou M, and Wang Z (2012) Notoginsenoside $\mathrm{Ft} 1$ promotes angiogenesis via HIF-1 $\alpha$ mediated VEGF secretion and the regulation of PI3K/AKT and Raf/MEK/ERK signaling pathways. Biochem Pharmacol 84:784-792.

Trautmann A, Toksoy A, Engelhardt E, Bröcker EB, and Gillitzer R (2000) Mast cell involvement in normal human skin wound healing: expression of monocyte chemoattractant protein- 1 is correlated with recruitment of mast cells which synthesize interleukin-4 in vivo. J Pathol 190:100-106.

Wang X, Ge J, Tredget EE, and Wu Y (2013) The mouse excisional wound splinting model, including applications for stem cell transplantation. Nat Protoc 8:302-309.

Address correspondence to: $\mathrm{Dr}$. Xiaojun Wu, Institute of Chinese Materia Medica, Shanghai University of Traditional Chinese Medicine, 201203, Shanghai, P.R.China. E-mail: xiaojunwu320@126.com; or Dr. Zhengtao Wang, Institute of Chinese Materia Medica, Shanghai University of Traditional Chinese Medicine, 201203, Shanghai, P.R.China. E-mail: wangzht@shutcm. edu.cn 